\title{
Optical Chiral Induced Spin Selectivity XMCD Study
}

\author{
Oren Ben Dora, Shira Yochelis ${ }^{\mathrm{a}}$, Hendrik Ohldag ${ }^{\mathrm{b}}$, and Yossi Paltiel ${ }^{\star_{\mathrm{a}}}$
}

\begin{abstract}
The chiral induced spin selectivity (CISS) effect in which selective transport of electron spins through helical chiral molecules occurs, has attracted a lot of attention in recent years. This effect was used to magnetize ferromagnetic (FM) samples by utilizing adsorbed chiral molecules. The electron transfer through the molecules was generated optically or electrically. In the optical configuration, circularly polarized light induced efficient magnetization by spin torque transfer (STT), using a hybrid of quantum dots (QDs) and chiral molecule self-assembled monolayer (SAM). Here, we use X-ray magnetic chiral dichroism (XMCD) spectroscopy in order to probe the optically induced magnetization on thin FM films. The results show differences in the FM magnetization depending on the optical circular polarization, matching previous non-local Hall probe measurements.
\end{abstract}

Keywords: Chiral induced spin selectivity · Magnetic chiral dichroism · Quantum dots · Spintronics
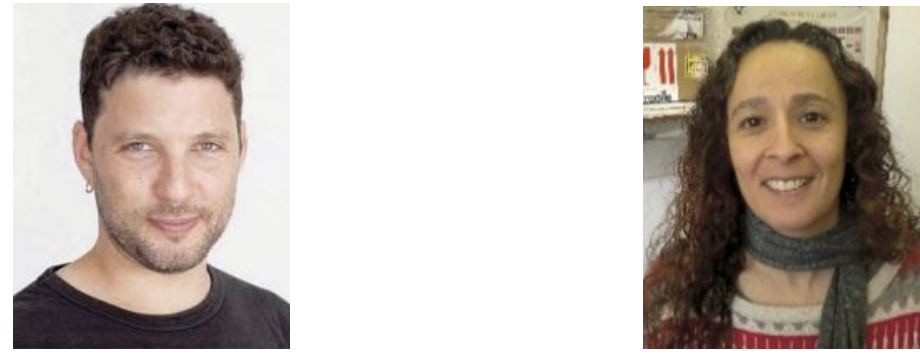

Oren Ben Dor studied Physics and received his BSc from Tel-Aviv University. He completed his MSc and $\mathrm{PhD}$ in the Applied Physics department in the Hebrew University of Jerusalem, in which he studied chiral induced spin selectivity phenomena occurring whenever spin specific electrons are transferred through a selfassembled monolayer of chiral molecules. He is currently conducting his postdoctoral research at the Center for Astrophysics at Harvard University, under supervision of Prof. Ronald Walsworth, researching neural network electrical behavior made possible by magnetic sensing by nitrogenvacancy centers in diamonds. He is married to Liron and the proud father of three beautiful daughters.
Shira Yochelis received her MSc and $\mathrm{PhD}$ in chemistry from The Weizmann Institute of Science, Israel in 2000 and 2005, respectively. Following work at InnoSense, Torrance, California, on hydrogen detectors, she joined in 2009 the group of Yossi Paltiel at the Applied Physics Department, Hebrew University of Jerusalem, as a research associate. She is involved in all aspects of group's activities and particularly responsible for the chemical research, including independent research.

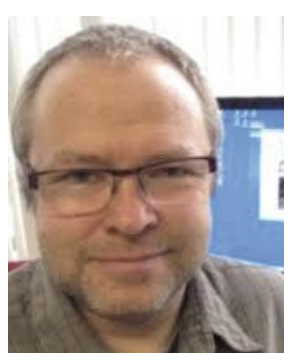

Hendrik Ohldag received his $\mathrm{PhD}$ in experimental physics from the Universität Düsseldorf, Germany, in 2002. He joined the Stanford Synchrotron Radiation Light Source (SSRL) in 1999 as a research assistant as part of his $\mathrm{PhD}$ research. After a postdoctoral fellowship at SSRL he became a permanent member of the research staff in 2005. Between 1999 and 2002 he was a visiting researcher at the Advanced Light Source (ALS) at Berkeley National Laboratory. Since 2014 he is a visiting researcher at New York University. Hendrik is a Staff Scientist in the X-ray Laboratory for Advanced Materials (XLAM) at the Stanford Synchrotron Radiation Laboratory, USA. In his research, he is studying exchange coupling phenomena between antiferromagnetic and ferromagnetic materials using polarization dependent X-ray absorption spectromicroscopy.

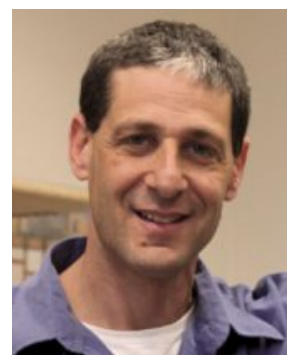

Professor Yossi Paltiel is now in the Applied Physics Department, the Hebrew University of Jerusalem Israel. Prof. Paltiel has worked for both high-tech industry groups and in the academic world. Since July 2009, he is leading the Quantum Nano Engineering group. Paltiel's group's goal is to establish a way to incorporate quantum mechanics into room temperature 'classical' computation and reading schemes. This will provide quantum coherence control at nanometer scale distances, while maintaining the physical characteristics of currently available computer input-output devices. Prof. Paltiel has published more 
than 100 papers in leading journals as well as issued 13 patents. $\mathrm{He}$ is also the co-founder of a startup company named Valentis NanoTech.

\section{Introduction}

X-ray magnetic chiral dichroism (XMCD) spectroscopy is extremely useful when attempting to detect minute local imbalances between spin up and spin down density of states (DOS), in a ferromagnetic (FM) thin film. These imbalances correspond to opposite $\mathrm{X}$-ray radiation circular polarizations in a fixed configuration, relative to the sample's normal to surface. X-ray circularly polarized beams, produced in a synchrotron facility, excite photoelectrons in the FM material from a core shell level to the Fermi level. Spinorbit coupling enables two different $2 p-3 d$ transitions, accounting for $2 p_{3 / 2}$ and $2 p_{1 / 2}$ levels, corresponding to $L_{3}$ edge absorption $(l+s)$ and $L_{2}$ edge absorption $(l-s)$ accordingly. The polarized photoelectrons that are excited from the core valance levels can occupy only empty conductivity band states in the $3 d$ shell according to their spin state. Selection rules allow for spin up photoelectrons to occupy only the empty spin up states, whereas spin down photoelectrons can occupy only the spin down empty states. Hence, the spin-split valence shell acts as a detector for the spins of the excited photoelectrons and the transition intensity is simply proportional to the number of empty $d$ states of a given spin. ${ }^{[1]}$

It is well established that a self-assembled monolayer (SAM) of chiral molecules is spin-selective whenever charges are transported through the layer, ${ }^{[2]}$ namely the chiral induced spin-selectivity effect (CISS). Hence, spins traversing in and out of the chiral SAM, adsorbed covalently on a FM substrate, can induce magnetic anisotropy (MA) in the FM thin layer. ${ }^{[3]}$ Only recently, a light-induced magnetization mechanism was introduced, combining QDs and a chiral SAM of Alpha Helix Polyalanine-L (AHPA-L) molecules that were chemisorbed on top of a thin FM film. By using circularly polarized light, that excited spin polarized electrons in the QDs, local optical magnetization was achieved. ${ }^{[4]}$ The excited spin delocalization relies upon the helical properties of the molecules. Therefore, the polarized electrons' probability to transfer through the chiral monolayer depends on the light polarization. A right-handed spin would propagate through a right-handed helical molecule with higher probability than a left-handed spin. This mechanism was probed by various Hall configurations and also by magnetic force microscopy
(MFM). Other light-induced studies have also shown similar behaviors. ${ }^{[5,6]}$

In this work, we probed the light-induced MA in a similar configuration using $\mathrm{X}$-ray magnetic chiral dichroism (XMCD) spectroscopy (Fig. 1). Here, a thin FM Ni film is deposited onto transmission electron microscopy (TEM) grids, with chiral SAM adsorbed on top linked covalently to colloidal QDs. Samples were simultaneously illuminated with a circularly polarized laser, used for spin excitation in the QDs, and a circularly polarized X-ray beam, used for MA detection. The results are consistent with previous experiments. This method enables to probe locally the magnetization and in principle study dynamics of CISS based spin injection.

\section{Experimental Details and Methods}

We used ultra-thin holey carbon TEM grid 400 mesh (Electron Microscopy Sciences) on which we deposited $5 \mathrm{~nm}$ of $\mathrm{Ni}$ using the e-beam evaporation method at room temperature (RT). Following deposition, TEM grids were left in an inert environment during all preparation processes. To prevent contact that could damage the delicate samples, $5 \mu \mathrm{l}$ of $1 \mathrm{mM}$ AHPA-L molecules in an ethanolic solution were dropcasted on top of the Ni films and left to dry. ${ }^{[7,8]}$ In the following step, $5 \mu$ l of toluene solution containing CdSe QDs was also dropcasted onto the samples and later rinsed in pure toluene. TEM grids were then packed in a sealed inert environment and sent to the Stanford Synchrotron Radiation Lightsource (SSRL) for the XMCD measurements. In order to test the magnetic properties of the bare $5 \mathrm{~nm}$ Ni layer, we used magnetic force microscopy (MFM), measuring magnetization on a selectively evaporated thin layer on a $\mathrm{SiO}_{2}$ substrate. The exposed Ni film showed strong magnetic behavior manifested by phase change in the MFM measurement (Figs. 2a,b). To study magnetization under illumination we prepared a non-magnetized thin film with Hall configuration. The anomalous Hall resistance $R_{x y}$ was measured under illumination on the bare $5 \mathrm{~nm} \mathrm{Ni}$ channel while a circularly polarized $532 \mathrm{~nm} \mathrm{cw}$ laser illuminated the sample with a power of $25 \mathrm{~mW}$ for $2 \mathrm{~min}$. A change of $\sim 0.7 \%$ in $R_{x y}$ was observed for $I_{x x}=1 \mathrm{~mA}$ current (Fig. 2c). In our XMCD experiments the laser power was an order of magnitude lower, showing that the chances for significant heating or other artefacts due to laser illumination on a bare sample is low.

The X-ray dichroism and absorption data were taken using the elliptical undulator beamline 13-1 at SSRL. This beamline provides X-rays with an energy between $250 \mathrm{eV}$ and $1250 \mathrm{eV}$ with a typical resolution of $0.1 \mathrm{eV}$. The polarization can be chosen freely between circular polarization of either helicity or linear vertical/ horizontal. The typical X-ray flux provided to the sample at this beamline is of the order of $10^{11}$ photons/sec. The beamline is equipped with a scanning transmission a.

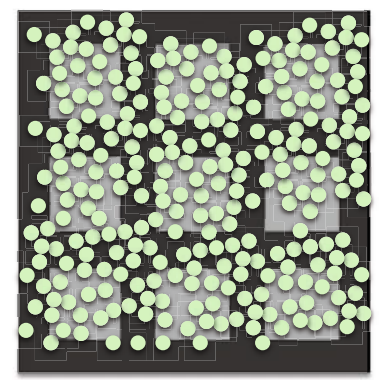

b.

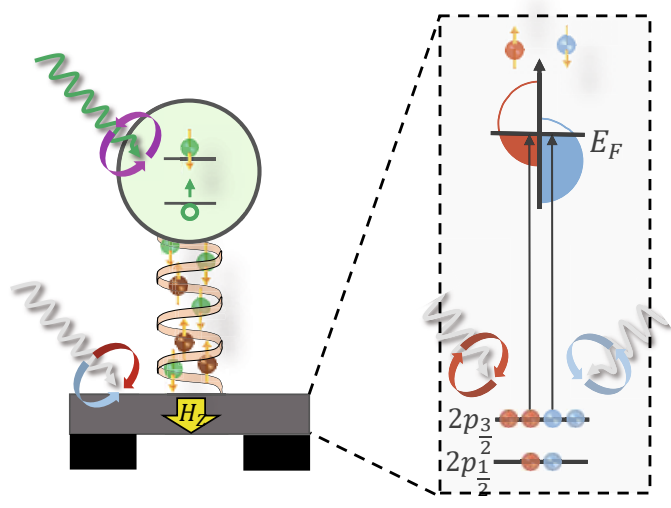

Fig. 1. XMCD experimental setup. a. Sample top view, $5 \mathrm{~nm}$ of suspended Ni film were deposited on top of the TEM grid, to which AHPA-L chiral SAM was adsorbed, linked to CdSe QDs (light green circles). b. Circularly polarized light (green wave) excites spin specific electrons (green circles) in the CdSe QDs (light green circle), hereby enabling propagation of specific spins through the AHPA-L chiral SAM and into the thin FM Ni film (grey rectangle), suspended on top of the TEM grid (black rectangles). An opposite moving spin specific electron (brown circles) propagates back to the QD to balance the now missing charge. Both downward and upward moving charges contribute to the same MA in the Ni. Right blowout: Different absorptions of the synchrotron-induced circularly polarized X-ray light, correspond to different spin up and spin down DOS near Fermi level (red and blue accordingly). The differences in DOS is translated, by the chiral layer, to MA in the thin Ni film. 

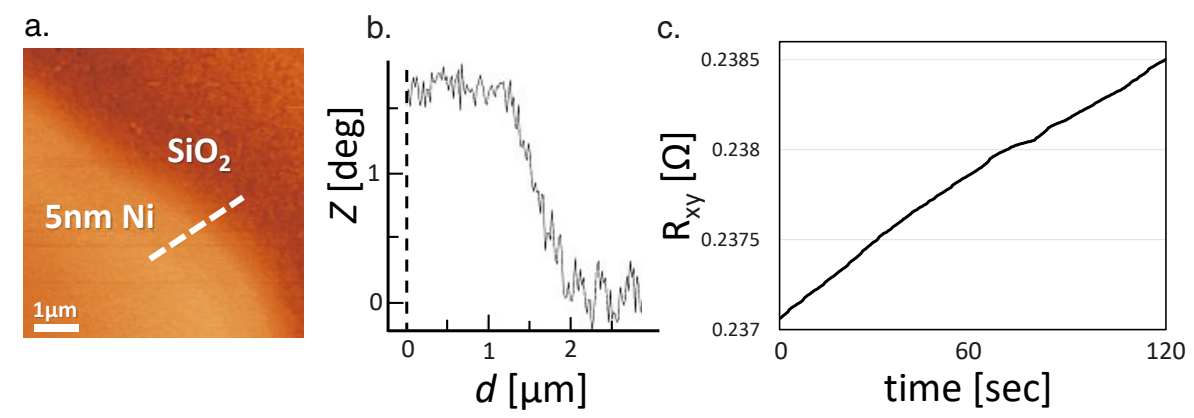

Fig. 2. Magnetic force microscopy and Hall effect characterization. a. Magnetic phase image made by magnetic force microscopy of $5 \mathrm{~nm}$ of $\mathrm{Ni}$ deposited on $\mathrm{SiO}_{2}$. b. Cross sectional view of the magnetic phase corresponding to dashed white line in (a). c. Hall resistance Rxy changes by $\sim 0.7 \%$ during continuous illumination for $2 \mathrm{~min}$ by a circularly polarized $532 \mathrm{~nm}$ cw laser with $25 \mathrm{~mW}$ power on a bare $5 \mathrm{~nm} \mathrm{Ni}$ deposited on a $\mathrm{SiO}_{2}$ substrate.

$\mathrm{X}$-ray microscope with a spatial resolution of about $30 \mathrm{~nm}$ using Fresnel zone plates, but it can generally be operated in a spectromicroscopy as well as a pure spectroscopy mode by removing the X-ray optics. The microscope operates at a pressure of $10^{-7}$ mbar or better.

\section{Results}

Upon arrival at SSRL the samples were first characterized under the microscope to evaluate the optimal way to go forward. Figs 3a-f shows scanning transmission X-ray microscopy (STXM) images acquired in transmission mode (left a,b,c) and electron yield mode (right d,e,f), with different field of views. The transmission images were acquired using a photo diode downstream of the sample, while the electron yield images were acquired by mounting the sample on an isolated holder. X-rays are absorbed in the Ni-layer, leading to the emission of a cascade of secondary electrons. To remain electrically neutral the sample has to be grounded, which is achieved by connecting it to an amperemeter capable of detecting picoampere currents. The magnitude of the current is then directly proportional to the X-ray absorption cross section. While the transmission yield detection is bulk sensitive, the electron yield detection is a surface sensitive approach with an exponential decay length of the electron yield of about $2-3 \mathrm{~nm}$ in metals, making it less suitable for our purposes. The transmission data in Figs $3 \mathrm{a}-\mathrm{c}$ shows that the Ni film thickness is quite homogeneous except for a small dust particle on the lower right side of the grid. The electron yield data does show some variation, however. In the following we acquired absorption spectra using the two methods and found that the transmission $L_{3}$ absorption spectrum resembles a clean Ni metal film, while the electron yield data shows an extra shoulder at $856 \mathrm{eV}$ as well as a general shift to higher photon energy, which is indicative of the presence of $\mathrm{NiO}$. We would like to point out that preliminary magnetic phase MFM measurements made on $5 \mathrm{~nm}$ Ni film deposited on $\mathrm{SiO}_{2}$ showed clear detectable magnetic behavior despite the apparent $\mathrm{NiO}$ layer, which is characterized by antiferromagnetic behavior (Figs 2a,b). The anomalous Hall resistance $R_{r,}$ measurements clearly show a negligible response to circularly polarized light, applied with an order of magnitude stronger power than that used in the XMCD experiments (Fig. $2 \mathrm{c}$ ), relieving any possibility of side effects that could overcome the underlined MA caused by the CISS effect. For this reason, we conclude that while the surface of the Ni films is slightly oxidized, it should not affect our synchrotron measurements. However, to avoid possible complications and because transmission yield data is less susceptible to external electric and mag- netic fields we decided to continue using the transmission detection.

In what follows, we removed the X-ray optics and mounted the sample under an angle of 45 degree with respect to the direction of the incoming X-rays. We magnetized the sample with a permanent magnet parallel to the X-rays, since XMCD is sensitive to the magnetization component parallel to the propagation direction of the $\mathrm{X}$-rays. In this geometry magnetization and X-rays thus include an angle of 45 degree. A $532 \mathrm{~nm} \mathrm{cw}$-laser with a power of 1-2 $\mathrm{mW}$ was installed at a right angle with respect to the X-ray direction. Both laser and X-ray beam were not focused so that the two spots of about $1 \times 1 \mathrm{~mm}^{2}$ in size could be easily overlapped. Finally, the polarization of the laser could be changed using an appropriate quarter wave plate.

An indication of the CISS effect, originating from the circular polarized light, is presented in Fig. 4, which shows Ni XMCD spectra acquired for different relative settings of the X-ray and laser polarization. In general, it is apparent that the circularly polarized laser radiation is able to change the size of the XMCD by about 5\% and hence the magnetic moment of Ni. While this effect is significant, it is too small to be reliably detected at the $L$, which is why we only show the $L_{3}$ resonance in the figure. These small differences are attributed to different spin specific charge transfer probabilities through the chiral AHPA-L SAM. When the X-ray beam is (+)-circularly polarized, transmission amplitude for (-)-laser polarization is $5 \%$ larger than that of the (+)-laser polarization. This higher transmission amplitude occurs as a result of higher $3 d(-)$ spin DOS occupancy, corre-

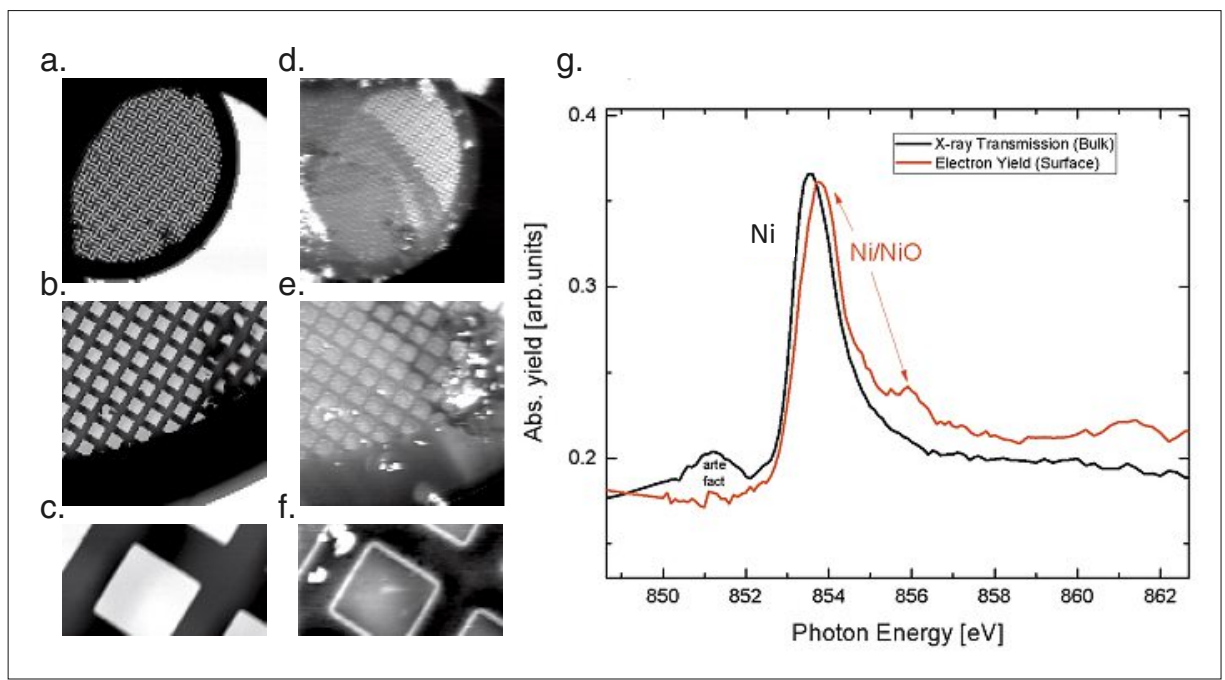

Fig. 3. Preliminary transmission yield measurements. X-ray transmission yield images of $2500 \times 2500 \mu \mathrm{m}^{2}$ and $100 \mu \mathrm{m}$ step (a), 600×600 $\mu \mathrm{m}^{2}$ and $20 \mu \mathrm{m}$ step (b), 80×40 $\mu \mathrm{m}^{2}$ and $1 \mu \mathrm{m}$ step (c), and their corresponding total electron yield images, where Ni film presence on top of the TEM grids was confirmed (d,e,f correspondingly). g. X-ray transmission, depicting bulk absorption, and total electron yield, depicting surface absorption, verified a $5 \mathrm{~nm}$ Ni layer and clean Ni bulk, alongside small native oxide presence in Ni layer. 


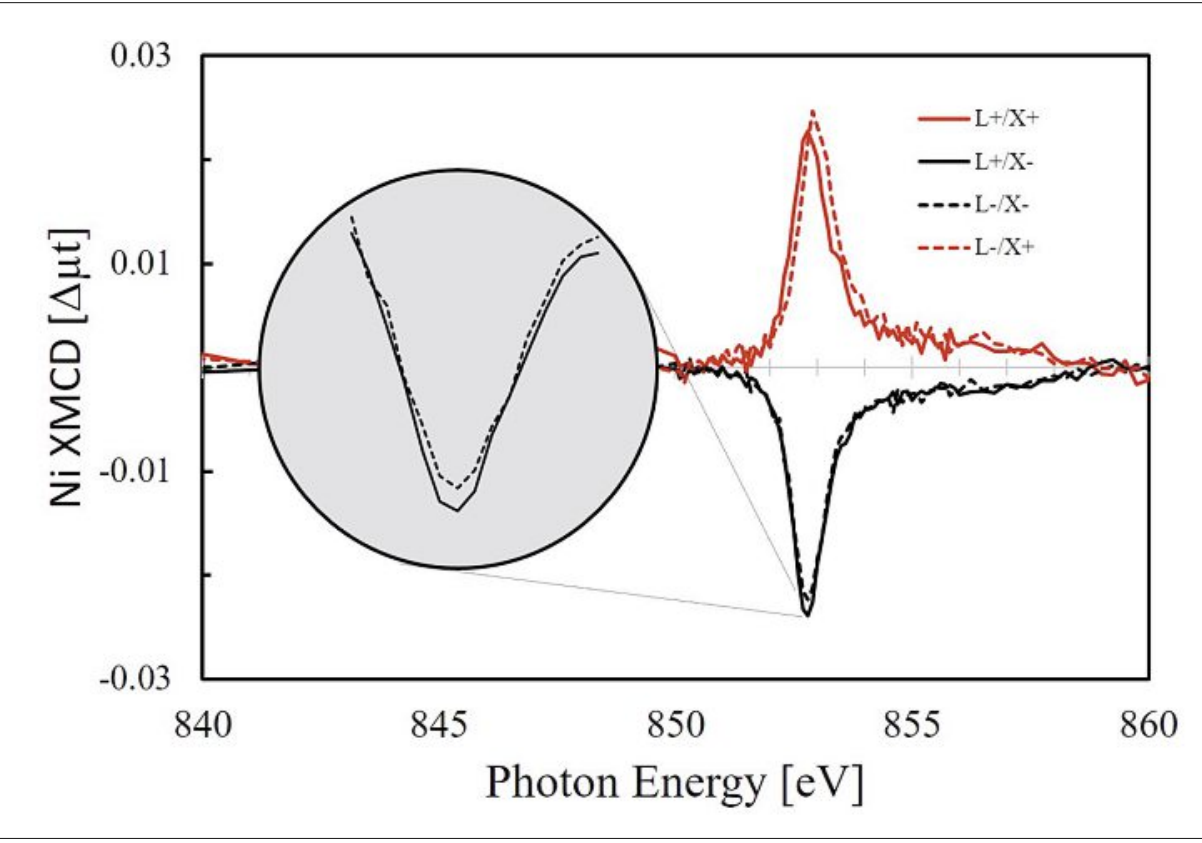

Fig. 4. XMCD transmission spectrum for CISS configuration. Circularly polarized laser light (depicted as L+ or L-) was used to excite the CdSe QDs. Circularly polarized X-ray beams (depicted as $\mathrm{X}+$ or $\mathrm{X}-$ ) excited core level electrons in the $L_{3}$ band edge clearly showing different transmission yields. These transmission yield differences point to an imbalance in the spin DOS, hence, different spin specific charge transfer probabilities through the chiral AHPA-L SAM corresponding to different laser circular polarizations. $L_{2}$ band edge absorption results are too noisy, perhaps due to spin injection which is centered at $E_{\mathrm{P}}$ and therefore does not convey any significant information and is not presented. The circular insert shows an enlarged image of the lower peaks.

sponding to (-)-laser polarization illumination. The opposite $3 d(+)$ spin DOS was not fully occupied, manifested as lower transmission amplitude, therefore larger absorption by core level $2 p_{3 / 2}$ spin (+) electrons. As expected, when the opposite X-ray beam (-)-polarization is used to probe the spin DOS, the opposite occurs, in which higher transmission is registered when $(+)$-laser polarization is applied and lower transmission is seen for (-)-laser polarization. Transmission spectrum in the $L_{2}$ band edge is noisy, perhaps due to spin injection which is centered at $E_{F}$ and consequently does not represent any significant data.

\section{Discussion}

The transmission yield differences between the two green laser circular polarizations' results, observed in the XMCD measurements, provide evidence for the CISS charge transfer mechanism. No external magnetic fields were used during data acquisition, so that the detected XMCD spectra are a direct measure of the magnetization of the Ni layer. It therefore appears as if the change or modulation of the magnetization was induced by the spin specific charge transfer through the chiral molecules. As mentioned elsewhere, ${ }^{[4]}$ the longitudinal spin coherence time, $\mathrm{T}_{1}$, is longer than typical transport times, thereby allowing spin injection into the FM layer through the AHPA-L SAM, resulting in the FM layer's magnetization reorienting outof-plane (OOP) via STT. This effect is enhanced by charge oscillation between the Ni layer and the QDs. ${ }^{[9]}$ Magnetization is sustained by this oscillation regardless of the current direction. ${ }^{[3,4]}$ Therefore, when circularly polarized light excites spins that correspond to the helical properties of the molecules, MA is expected to occur with higher probability than when the opposite circular polarization is used. Once majority spin DOS is highly populated, the circularly polarized X-ray light, probing the majority spin states, yields higher transmission yield. This is because there are less core level $2 p$ electrons for this spin DOS, due to $2 p-3 d$ transitions.

By using the anomalous Hall effect (AHE) (Fig. 2c) measurements we demonstrate that heating is negligible in our experiment. Heating solely does not break the Hall symmetry and is not expected to induce the Hall effect. Moreover, heating is not linear, while the Hall signal is expected to grow with time due to the domain magnetization ordering process. In Fig. 2c we present a linear dependency between the laser time and the Hall voltage. Furthermore, both right and left laser circular polarizations were used with the same heating power giving opposite results in Hall voltage, suggesting that a large part of the change in XMCD signal comes from different spin polarizations within the lat- tice resulting from the spin-specific charge transfer mechanism reported here.

Several reasons might contribute to the small 5\% transmission yield difference between the two laser circular polarizations' effects. We used polycrystalline $\mathrm{Ni}$ film that was deposited by e-beam at RT. This evaporation technique yields film with degraded polarizability. First the laser power was set to a maximum of $1-2 \mathrm{~mW}$, instead of $10 \mathrm{~mW}$ used in the former experiment. More importantly, the lifetime of the spin in the $\mathrm{Ni}$ films is smaller, dramatically reducing the MA strength. The similarity between transmission yields of (-) and (+) $\mathrm{X}$-ray circular polarized beams can be reasoned by the fact that $\mathrm{Ni}$ is characterized by a small spin up and down DOS difference near Fermi level. ${ }^{[10]}$ Such small differences are hard to reveal when polarization is small to begin with. The relative shift in energy between the two red curves is attributed to spin-lattice relaxation mechanisms. Spin-flip excitations occurring on the ps time scale can alter the $3 \mathrm{~d}-4 \mathrm{sp}$ hybridization of the $\mathrm{Ni}$, and hence alter the bandwidth, which alternatively changes the relative $E_{f}$ location with respect to the core electrons, manifested as an energy shift. ${ }^{[1-13]}$ This energy shift cannot be related to heating, as both laser circular polarizations were performed with the same power. In our device spin-lattice relaxation occurs on a ps time scale, explaining very well the observed shift.

Also, several studies have shown that for disordered thin $\mathrm{Ni}$ films polarizations reach a maximum of $\sim 14 \%$. ${ }^{[14-16]}$ Moreover, both incident X-ray beam and circularly polarized laser used to excite the QDs were tilted at an angle of $45^{\circ}$, further reducing spin polarization by an added cosine factor. Lastly, the AHPA-L chiral molecules were dropcasted instead of several hours of sample immersion. This creates a less organized monolayer, though still robust, ${ }^{[17]}$ which manifests as lower surface molecular concentration and larger tilting angles between molecules and normal to surface, ${ }^{[18,19]}$ thereby further reducing the injected spins' polarization effect onto the $\mathrm{Ni}$ film.

Nevertheless, the signal is large enough to study spin dynamics when the opticallyoriginated spin injection results from CISS effect. Using a more focused X-ray beam, local spin distribution injected from selective adsorbed molecules can be probed.[20]

\section{Summary}

Here we show XMCD measurement results of light-induced magnetization. By illuminating circularly polarized light on QDs attached to a chiral SAM that is adsorbed on thin FM Ni film, spin torque 
is transferred to and from the FM, thus reorienting its magnetic dipole. We found that opposite circular polarization magnetization outcomes, visualized by XMCD spectroscopy, differed by $5 \%$. This could be explained by taking into account our device configuration together with other technical constraints. The results described here lie in good agreement with previous results, reaffirming the use of this system to study dynamics of the optically-induced spin injection.

\section{Acknowledgements}

YP acknowledges the support of the Israel Science Foundation grant \#273/15 and the Ministry of Science Israel. OBD would also like to acknowledge the Israeli Ministry of Science, Technology and Space grant \#0399174. YP and OBD wish to thank the Stanford Synchrotron Radiation Lightsource facility for performing the XMCD measurements. Use of the Stanford Synchrotron Radiation Lightsource, SLAC National Accelerator Laboratory, is supported by the U.S. Department of Energy, Office of Science, Office of Basic Energy Sciences under Contract No. DE-AC02-76SF00515.

Received: March 29, 2018
[1] J. Stöhr, J. Magn. Magn. Mater. 1999, 200, 470.

[2] R. Naaman, D. H. Waldeck, J. Phys. Chem. Lett. 2012, 3, 2178.

[3] O. Ben Dor, S. Yochelis, S. P. Mathew, R. Naaman, Y. Paltiel, Nat. Commun. 2013, 4, 2256.

[4] O. Ben Dor, N. Morali, S. Yochelis, L. T. Baczewski, Y. Paltiel, Nano Lett. 2014, 14, 6042.

[5] K. S. Kumar, N. Kantor-Uriel, S. Pulinthanathu Mathew, R. Guliamov, R. Naaman, Phys. Chem. Chem. Phys. 2013, 15, 18357.

[6] P. C. Mondal, P. Roy, D. Kim, E. E. Fullerton, H. Cohen, R. Naaman, Nano Lett. 2016, 16, 2806.

[7] J. Chen, I. Ratera, A. Murphy, D. F. Ogletree, J. M. J. Fréchet, M. Salmeron, Surf. Sci. 2006, 600, 4008.

[8] M. Mottaghi, P. Lang, F. Rodriguez, A. Rumyantseva, A. Yassar, G. Horowitz, S. Lenfant, D. Tondelier, D. Vuillaume, $A d v$. Funct. Mater. 2007, 17, 597.

[9] M. Eckshtain-Levi, E. Capua, S. RefaelyAbramson, S. Sarkar, Y. Gavrilov, S. P. Mathew, Y. Paltiel, Y. Levy, L. Kronik, R. Naaman, Nat. Commun. 2016, 7, 10744

[10] J. Tersoff, L. M. Falicov, Phys. Rev. B 1982, 26, 6186.

[11] C. Stamm, T. Kachel, N. Pontius, R. Mitzner, T. Quast, K. Holldack, S. Khan, C. Lupulescu, E. F. Aziz, M. Wietstruk, H. A. Dürr, W. Eberhardt, Nat. Mater. 2007, 6, 740.

[12] K. Carva, D. Legut, P. M. Oppeneer, EPL Europhys. Lett. 2009, 86, 57002.
[13] T. Kachel, N. Pontius, C. Stamm, M. Wietstruk, E. F. Aziz, H. A. Dür, W. E. Eberhardt, F. M. F. de Groot, Phys. Rev. B 2009, 80, 092404.

[14] C. Rau, S. Eichner, Phys. Rev. Lett. 1981, 47, 939.

[15] U. Gradmann, Appl. Phys. 1974, 3, 161.

[16] P. M. Tedrow, R. Meservey, Phys. Rev. Lett. 1971, 26, 192.

[17] O. Ben Dor, S. Yochelis, A. Radko, K. Vankayala, E. Capua, A. Capua, S.-H. Yang, L. T. Baczewski, S. S. Papworth Parkin, R. Naaman, Y. Paltiel, Nat. Commun. 2017, 8 , 14567.

[18] N. Peer, I. Dujovne, S. Yochelis, Y. Paltiel, ACS Photonics 2015, 2, 1476

[19] S. Sek, A. Tolak, A. Misicka, B. Palys, R Bilewicz, J. Phys. Chem. B 2005, 109, 18433.

[20] Y. Shiratsuchi, Y. Kotani, S. Yoshida, Y Yoshikawa, K. Toyoki, A. Kobane, R. Nakatani, T. Nakamura, Mater. 2015, 2, 484. 